\title{
Reproducción de cochinilla silvestre Dactylopius opuntiae (Homóptera: Dactylopiidae)
}

\section{Reproduction of wild cochineal Dactylopius opuntiae (Homoptera: Dactylopiidae)}

\author{
Arnoldo Flores-Hernández², Bernardo Murillo-Amador ${ }^{1 *}$, Edgar Omar Rueda-Puente ${ }^{3}$, José Cruz Salazar-Torres ${ }^{4}$, \\ José Luis García-Hernández ${ }^{1}$ y Enrique Troyo-Diéguez ${ }^{1}$ \\ ${ }^{1}$ Centro de Investigaciones Biológicas del Noroeste, S.C., Mar Bermejo 195, 23090.Col. Playa Palo de Santa Rita. La Paz, Baja California Sur, México. \\ *Correspondencia: bmurillo04@cibnor.mx \\ ${ }^{2}$ Unidad Regional Universitaria de Zonas Áridas-Universidad Autónoma Chapingo, Bermejillo, Durango, México. \\ ${ }^{3}$ Departamento de Administración Agropecuaria-Universidad de Sonora, Santa Ana, Sonora, México. \\ ${ }^{4}$ Departamento de Parasitología Agrícola-Universidad Autónoma Chapingo, Estado de México.
}

Resumen. Por sus características, la cochinilla silvestre ofrece perspectivas de aprovechamiento como fuente de carmín y para el mejoramiento genético de la grana fina. Por lo anterior, se estableció una multiplicación y crianza de cochinilla silvestre, proveniente de la zona árida del norte de México para caracterizarla, utilizando como hospedero a Opuntia megacantha Salm Dyck. Los resultados indican que la especie silvestre pertenece al género-especie Dactylopius opuntia. Se determinó la presencia de partenogénesis en hembras. La duración de los estadios biológicos depende del sexo del insecto; el adulto hembra permaneció durante 38.4 días y los machos 4.2 días, mientras que los primeros estadios ninfales duraron 18.1-19.8 días. El ciclo biológico de las hembras fue de 77 días mientras que el de los machos fue de 43 días. Para las hembras se estimó un periodo de preoviposición de 18.8 días, manteniéndose en oviposición durante 21 días con un promedio de 131 insectos por hembra. La proporción sexual hembras: machos fue 1:1. El tipo de reproducción fue predominantemente sexual, aunque hubo hembras partenogénicas. Este es el primer reporte de Dactylopius opuntiae como cochinilla silvestre asociada a la zona árida del noreste de México, específicamente en el Bolsón de Mapimí, Durango, México.

Palabras clave: Dactylopius opuntiae, nopal, Opuntia spp., tinte carmín, sobrevivencia, reproducción.

\begin{abstract}
Wild cochineal has characteristics that offer advantage as a source of carmine and for fine cochineal improvement. To characterize wild cochineal, we initiated a breeding effort raising wild cochineal from the arid zone of the north of Mexico, using as a host organism Opuntia megacantha Salm Dyck. The results indicate that the wild species is Dactylopius opuntiae. The presence of parthenogenesis in females was determined. The duration of ontogenetic stages depends on the sex of the insect. The adult female lasted 38.4 days and 4.2 days for males; the first stage nymphs were similar in duration (18-19.8 days). The complete biological cycle of the females was 77 days, and in the males 43 days. A period of preoviposition of 18.8 days for the females was found, laying eggs during 21 days with an average of 131 insects per female. The sex ratio female: male was 1:1. The reproduction generally was sexual although there were parthenogenetic females. This is the first report of Dactylopius opuntiae as a source of wild cochineal in the arid zone of North, Central and Northwest Mexico, specifically in the Bolson of Mapimí, Durango, Mexico.
\end{abstract}

Key words: Dactylopius opuntiae, prickly pear, Opuntia spp., carmine dye, survival, reproduction.

\section{Introducción}

Desde tiempos antiguos se han distinguido dos tipos de cochinilla: la fina o cultivada y la silvestre o corriente. Ambas parasitan el género Opuntia y Nopalea (Ferris, 1955). Las hembras grávidas de grana fina se han utilizado como fuente para la obtención de carmín, colorante natural que ha

Recibido: 25 octubre 2004; aceptado: 17 enero 2006 recobrado importancia al descubrirse que los artificiales son agentes cancerígenos.

Sus aplicaciones son diversas en la industria cosmética, alimenticia, farmacéutica y textil, entre otras (Vigueras y Portillo, 1997). La grana fina es la cochinilla comercial; la silvestre, debido a la baja concentración y calidad del colorante, se utiliza esporádicamente. Sin embargo, sus características afines y distintivas ofrecen perspectivas de aprovechamiento como fuente del colorante carmín y para 
mejoramiento genético de la grana fina (Aquino, 1991). Asimismo, en algunos países sudafricanos tiene importancia económica por su utilidad como biocontrol de poblaciones de Opuntia (Morán y Zimmerman, 1991). En las zonas áridas del norte de México, específicamente en el Bolsón de Mapimí, algunas especies de cactus y plantaciones de nopales son afectadas por la cochinilla, la cual no ha sido apropiadamente estudiada y comienza a ser una plaga considerable (Cruz, 1990). Nuestro interés en este insecto silvestre es extender el conocimiento tomando en cuenta algunos aspectos biológicos para promover su reproducción natural .y su utilización.

Ferris (1955) ubica las cochinillas en la familia Dactylopiidae, dentro del cual se encuentra el género Dactylopius con 8 especies silvestres, además de la grana fina (De Lotto, 1974), con distribución mundial; taxonómicamente se considera uno de los insectos más difíciles de clasificar (Zimmermann, 1948). La biología de este tipo de escamas, como también se les nombra, es similar. No obstante, la información sobre aspectos biológicos importantes, como tipo de reproducción o duración del ciclo biológico, es muy variable. En estudios recientes, basados en información morfológica, de cariotipos, análisis cladísticos y moleculares, se sugiere que Dactylopius pertenece a Eriococcidae (Aquino, 1991; Gullan y Cook, 1998). Recientemente, Eriococcidae fue determinada ser no-monofilético y cada género requerirá una revisión sustentada en información sistemática, con datos biológicos, morfológicos y moleculares para generar hipótesis de la relación del número de grupos Ericoccoides, proponer nuevos grupos de clasificación, reconocer y describir nuevas especies (Cook y Gullan, 2004).

En cochinilla fina se han encontrado respuestas diferentes en cuanto al fenómeno de partenogénesis, que es muy común en la superfamilia Coccoidea a la cual pertenece. Además, la presencia de dicho fenómeno se ve influenciada por factores del medio, como la temperatura, que también tiene efectos importantes en la duración del ciclo biológico, proporción de sexos, supervivencia y mortalidad de diversas especies del género Dactylopius (Mann, 1969; Marín y Cisneros, 1977; Cruz, 1990; Santibañez, 1990; Méndez, 1992). En la cochinilla fina (D. coccus Costa), Méndez (1992) observa que cuando aumenta la temperatura la duración del ciclo tiende a disminuir. Sin embargo, la probabilidad de que los individuos lleguen al estado adulto es menor; se incrementa el establecimiento de ninfas y el número de machos tiende a disminuir. En D. austrinus, Hosking (1984) encontró que el número de huevecillos depositados por hembra fue mayor a temperaturas de $27.5^{\circ} \mathrm{C}$; a partir de ésta, disminuyó hasta alcanzar su punto más bajo a $34^{\circ} \mathrm{C}$. Hay teorías que explican que la determinación sexual en cóccidos puede alterarse por cambios en la condición fisiológica del huevo, como las causadas por temperaturas no óptimas y retraso en el apareamiento (Nur et al., 1987).

Entre los factores que influyen en el establecimiento de la grana se encuentran el hospedante y los depredadores o parásitos (Flores et al., 1985; Bátiz, 1987). Las características del hospedante que se consideran de importancia son la susceptibilidad, el estado nutrimental, la edad y la orientación del cladodio, (Marín, 1985; Lazos, 1987; Méndez et al., 1990; Montiel, 1992).

El objetivo de esta investigación fue la caracterización y el estudio del comportamiento en la reproducción de la cochinilla silvestre en zonas áridas, tales como de la parte central del noreste de México donde prevalecen condiciones climáticas secas y en las que este insecto no ha sido estudiado apropiadamente.

\section{Materiales y métodos}

Esta investigación se realizó en el laboratorio de insectos de la Unidad Regional Universitaria de Zonas ÁridasUniversidad Autónoma Chapingo (URUZA-UACh) en Bermejillo, Durango, México, bajo condiciones controladas de temperatura y luminosidad natural parcial.

Identificación taxonómica. Se colectaron cochinillas silvestres que se encontraban en plantas de nopal en el vivero de la URUZA y se preservaron en alcohol al 70\%. En el laboratorio se realizó el montaje con el método para cóccidos propuesto por Solís (1993), y para la identificación, que se llevó a cabo en el departamento de parasitología agrícolaUACh, se utilizó la nomenclatura de Ferris (1955), Howell y Williams (1976) y De Lotto (1974) que corresponden a familias, géneros y especies, respectivamente, y se examinaron bajo microscopio compuesto 73 laminillas de hembras adultas. Como segunda fase, se consideró la reproducción de los insectos de cochinilla colectados, con el fin de conocer los aspectos biológicos que posteriormente se describen.

Aspectos biológicos. En el otoño de 1999 se estableció un pie de cría y se obtuvieron 2 generaciones de cochinilla hasta el inicio del experimento en el mes de febrero de 2000. Como el hospedante se utilizaron cladodios de un año de edad del cultivar 68 de Opuntia megacantha, donde previamente se había observado mejor desarrollo del insecto, bajo la modalidad de pencas suspendidas. La cohorte inicial consistió de 111 insectos para determinar tablas de vida, duración de estadios, ciclo biológico, diferenciación y proporción sexual, preoviposición, inicio y periodo de oviposición. Para aislar a las ninfas se construyeron jaulas de tres diferentes tamaños con vasos de plástico, número cero (jaula tipo 1), número ocho (jaula tipo 2) y número 16 (jaula tipo 3 ), cerrados en un extremo con tela organza y adheridos a las pencas con silicón. La cohorte en conjunto fue aislada en jaulas tipo 2, que al día siguiente se retiraron al disminuir el movimiento de las ninfas. A partir de ese momento se realizaron conteos y observaciones cada tercer día llevando registro de cada 
individuo, utilizando microscopio estereoscopio o lupa de cuatro aumentos.

En la duración de estadios se consideró un diseño completamente al azar con 6 tratamientos en un arreglo factorial; el factor uno fue sexo (hembra y macho) y el dos, el estado biológico (ninfa I, ninfa II y adulto). La duración de estadios biológicos se determinó con las mudas; la proporción de hembras:machos, al momento de formación de cocones por parte de los machos y con el aumento en tamaño de las hembras. En el caso de aspectos propios de las hembras como el inicio de oviposición, se tomó con la aparición de la primera ninfa cercana a la madre; la preoviposición, con la diferencia del inicio de oviposición y el tiempo al que quedan grávidas las hembras. Finalmente, la oviposición se consideró del periodo de inicio a la aparición de la última ninfa ovipositada.

Para determinar la presencia de partenogénesis se utilizó un diseño experimental de bloques completos, al azar, con cuatro repeticiones de 20 hembras cada uno. Los tratamientos fueron tres y consistieron en hembras con adición de 2 machos; hembras sin adición de machos y hembras de apareamiento natural, como testigo. Los machos se colocaron antes de la emergencia del cocón. Al empezar la oviposición, las hembras fueron desprendidas de las pencas y colocadas en vasos de poliestireno número 114 para realizar conteos de la progenie de cada una. La partenogénesis y los parámetros poblacionales se calcularon de acuerdo con Vera et al. (1997); las diferencias mínimas significativas entre medias de tratamientos fueron separadas utilizando la prueba de rango múltiple de Duncan al 0.05. Todos los análisis estadísticos se realizaron utilizando el programa estadístico SAS (SAS, 1996).

\section{Resultados y discusión}

De acuerdo con las características indicadas por De Lotto (1974), la cochinilla silvestre distribuida en la región norte de México (Bermejillo, Durango) se identificó como Dactylopius opuntiae Cockerell, con base en los rasgos de la hembra adulta distinguibles en la región dorsal: setas con ápice truncado de 2 tamaños, presencia de algunos poros en el margen externo de los espiráculos torácicos posteriores y la agrupación de racimos en los últimos 3 segmentos abdominales, considerados por De Lotto (1974), Millar (1976) y Solís (1993). Las observaciones mostraron que las hembras son de metamorfosis incompleta y los machos de metamorfosis completa. Los instares ninfales son de color rojo que se diferencian por la secreción pulverulenta similar al talco y por la abundancia de filamentos blancos, así como por el aumento en tamaño conforme avanza su desarrollo.

Duración de estadios biológicos. Las observaciones en el experimento de la cochinilla silvestre distribuida en la región árida del norte de México mostró que las hembras de $D$. opuntiae tienen 3 estadios biológicos: huevo, ninfa (con 2 instares) y adulto; mientras que los machos mostraron la presencia de huevo, ninfa, prepupa, pupa y adulto. Estos resultados son similares a los de De Lotto (1974) y Miller (1976), quienes consideran incompleta la metamorfosis en las hembras, mientras que los machos aparentemente la tienen completa. Las ninfas nuevas fueron de color rojo y aproximadamente después de una semana expelieron una sustancia blanca filamentosa y una secreción también blanca, similar al talco. Después de la primera metamorfosis, el color del insecto fue oscureciéndose y su tamaño se incrementó lentamente; la secreción filamentosa y la pulverulenta fueron abundantes. En la segunda metamorfosis la piel nueva, producto de la muda, cubrió a la hembra adulta. La hembra adulta es grande, comparada con el máximo tamaño que alcanza antes de la oviposición. Cuando las hembras alcanzan la etapa adulta expulsan huevos, los cuales permanecen en la parte de abajo de su cuerpo. Estas observaciones son similares a las obtenidas con $D$. coccus y observadas por Alzate (1794), Piña (1977) y Marín y Cisneros (1977).

Los 2 primeros instares ninfales fueron de 18.1 y 19.8 días para hembras y machos, respectivamente, resultados similares a los que obtuvieron Velasco y García (1988) con D. coccus (14 a 19 días para el primer instar de la hembra y de 18 a 22 días para el macho). Sin embargo, Méndez (1992) estimó una duración de 31 días en promedio para el primer instar ninfal de la cochinilla fina o cultivada.

La duración del ciclo biológico en las hembras fue de 77 días y de 43.3 días para machos, similar a la estimada por Cruz (1990), quien estudió la grana fina y encontró que el adulto hembra dura entre 35 y 42 días y que el macho adulto es de vida efímera pues sólo vive 3 o 4 días (Fig. 1). Aunque los datos colectados son similares a los registrados, muchos autores están de acuerdo que la duración de los diferentes estadios biológicos es una función lineal de la temperatura (Cruz, 1990; Santibáñez, 1990; Velasco y García, 1990). En el presente trabajo, el promedio registrado de temperatura mínima fue de $19.5^{\circ} \mathrm{C}$ y el de la máxima, de $23.6^{\circ} \mathrm{C}$, entre los meses de febrero a abril de 2000 .

La diferenciación sexual del macho, 7 días después de la primera muda, emergió de manera paralela a la segunda muda en las hembras. El periodo estimado de oviposición fue de 18.8 días; se mantuvo por 21 días con un promedio de 131 huevos. Los resultados obtenidos se apoyan en los de Méndez (1992), quien afirma que en la grana cultivada existe cierta sincronización entre la emergencia del macho y la segunda muda de las hembras, que Marín y Cisneros (1977) señalan como el inicio de la receptividad en éstas. Proporción sexual. Los resultados no fueron significativos en la cantidad de hembras y machos $(F=0.16 ; \mathrm{gl}=1,6 ; P=0.7)$ generados por 7 hembras; se consideró una proporción sexual 1:1 (machos $=23$ y hembras $=24)$, similar a los datos que registró Cruz (1990) en cochinilla cultivada y Gilreath y Smith (1987) en D. confusus a los 22 y $26^{\circ} \mathrm{C}$. También Cruz (1990) encontró que la variabilidad en la proporción sexual puede deberse a factores como la alimentación, la técnica 


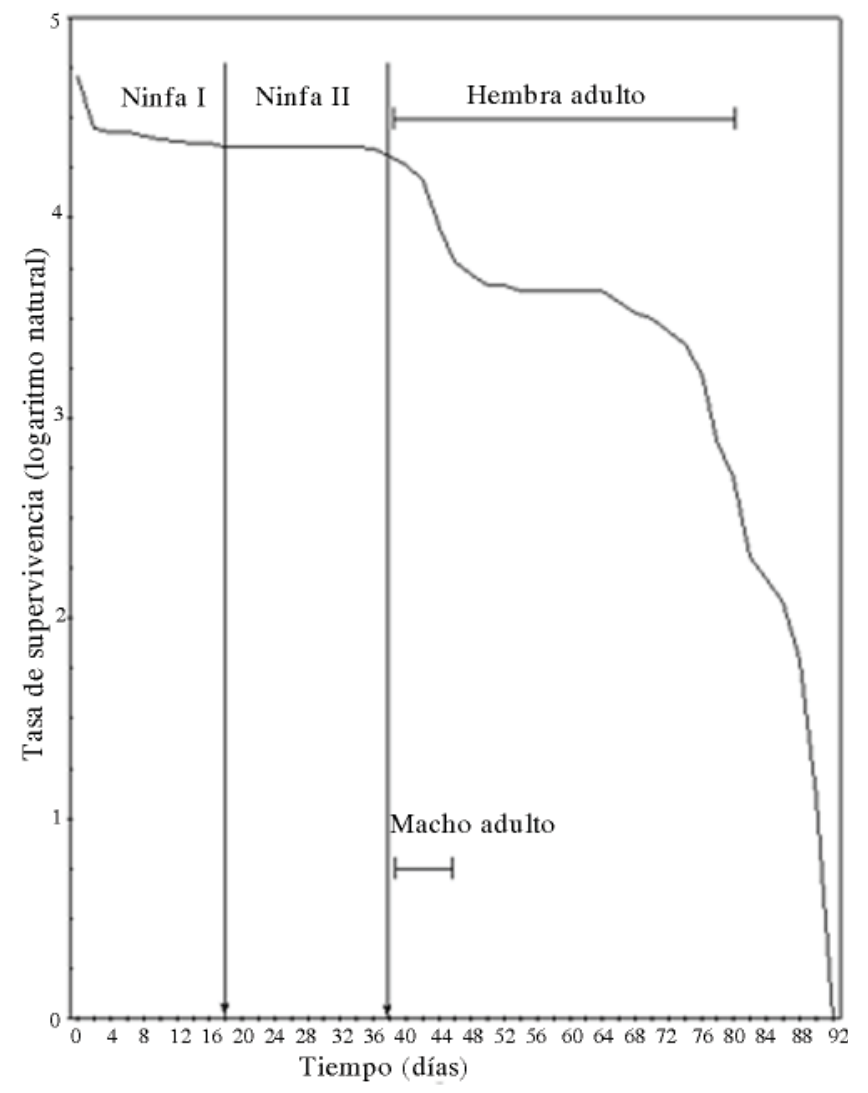

Figura 1. Curva de sobrevivencia de machos y hembras de Dactylopius opuntiae en cladodios de Opuntia megacantha SalmDick bajo condiciones semi-controladas en una región árida del norte de México (Bermejillo, Durango).

de infestación y el manejo, entre otros. Marín y Cisneros (1977), al estudiar D. coccus, encontraron que el número de hembras aumenta al pasar de condiciones de laboratorio a las de campo.

Reproducción. Los resultados mostraron diferencias significativas entre tratamientos $(F=25.82 ; \mathrm{gl}=2,234$; $P<0.0001)$ revelando que no se presenta el fenómeno de partenogénesis común en la superfamilia Coccoidea (Miller, 1976). Sin embargo, hubo descendencia en el tratamiento donde no se adicionaron machos, por lo que se consideran hembras partenogénicas. El promedio de insectos crías ovipositadas por las hembras a las que se les colocaron 2 machos fue de 154.8; las de apareamiento natural generaron 131.2 insectos, mientras que las que no tuvieron machos sólo tuvieron 61.8 insectos ovipositados. El máximo de insectos ovipositados fue 617 y se presentó en el tratamiento con 2 machos. Algunos autores han obtenido un fenómeno de partenogénesis con cambios lentos, modificando algunas condiciones del medio ambiente (temperatura y fotoperiodo), las cuales influyen en el desempeño de la reproducción partenogénica y pueden hacer variar la proporción sexual a lo largo de generaciones sucesivas (Davey, 1968).

Parámetros poblacionales y tablas de vida. El promedio de insectos que alcanzaron la etapa adulta fue del $64.8 \%$ para machos y $66.7 \%$ para hembras. Las causas posibles de la mortalidad alta en cochinilla fina o cultivada, de acuerdo con Gilreath y Smith (1987), Cruz (1990) y Méndez (1992) son los factores ambientales, como las temperaturas extremas y la falta de protección contra las mismas, ya que durante el primer estadio los individuos carecen de la capa cerosa que los cubre.

La mortalidad alta observada al inicio del ciclo del insecto y el movimiento que presentan las ninfas de $D$. Coccus durante sus diferentes etapas, los puede ocasionar, entre otras causas, el bajo nivel que aportan las especies sin espinas (como Opuntia megacantha), lo cual las hace poco atractivas al ataque de los insectos (Méndez, 1992); la respuesta de la planta al daño, causando la compresión del ápice de la proboscis mediante la expansión de las células epidermales ocasionando la muerte del insecto (Hamlin, 1932; Mann, 1969), o bien, mediante un efecto de antibiosis (Kogan, 1990); la presencia de una capa de oxalatos de calcio en la pared de las células epidermales (Trachtenberg y Mayer, 1982), que posiblemente impida la inserción de los estiletes; la influencia de la temperatura sobre la supervivencia y, por último, la gran variación genética que existe dentro del género Opuntia (Gilreath y Smith, 1987).

\section{Conclusiones}

El tipo de reproducción puede ser considerado sexual, debido a las diferencias altamente significativas entre la descendencia producida por las hembras con machos y del apareamiento natural en relación con las hembras sin machos. Sin embargo, algunas hembras fueron identificadas como partenogénicas. El promedio de la duración del ciclo biológico en hembras fue de 77 días y de 43.3 días en machos. Ambos instares en las hembras y machos duraron entre 18.1 y 19.8 días. La diferenciación sexual de los machos ocurrió 7 días después de la primera muda, en sincronía con el segundo cambio de las ninfas que originarían hembras y posiblemente con la madurez sexual de estas últimas. El periodo de la preoviposición se consideró de 18.8 días; el ciclo de oviposición permaneció 21 días con un promedio de 131 descendientes. De acuerdo con los datos anteriores, basados en las características de las hembras adultas de cochinilla silvestre distribuida en la localidad de Bermejillo, Durango, México, la biología de cochinilla silvestre es similar a la de cochinilla fina o cultivada Dactylopius opuntiae. Los resultados encontrados en este estudio muestran el potencial de la cochinilla silvestre en cuanto a su adaptación a las condiciones ambientales predominantes en la región y apoyan su utilización futura con fines de aprovechamiento.

Las similitudes en la biología de la especie silvestre con 
las de la fina hacen factible el mejoramiento genético de la especie cultivada; sin embargo, es necesario realizar estudios biológicos a través de generaciones sucesivas, dado que se ha visto que la biología del insecto es influenciada por las condiciones ambientales.

Este es el primer registro de cochinilla silvestre, Dactylopius opuntiae, asociada a la zona árida del norte, centro y noreste de México, donde las condiciones climáticas secas están presentes, específicamente en el Bolsón de Mapimí, Durango, México.

\section{Agradecimientos}

La presente investigación fue apoyada por la Unidad Regional Universitaria de Zonas Áridas-Universidad Autónoma Chapingo, Bermejillo, Durango, México(069/1999), por el proyecto ZA 1 del Centro de Investigaciones Biológicas del Noroeste, S.C. (CIBNOR) y por la Dirección General de Investigación y Posgrado-UACh.

\section{Literatura citada}

Alzate, J. A. 1794. Memoria del insecto grana o cochinilla (1777-1794). Gacetas de Literatura de México (Puebla) 3: 199-259.

Aquino, P. G. 1991. Estudio cromosómico en cuatro tipos de cochinilla (Dactylopius spp.) (Homóptera: Dactylopiidae) del nopal (Opuntia spp). Tesis de maestría, Colegio de Postgraduados,. Montecillo, Estado de México. 139 p.

Bátiz, M. A. 1987. Aspectos biológicos agronómicos y experiencias actuales en torno al cultivo del nopal y la cochinilla. In La grana y el carmín (inédito). México D.F., p. 89

Cruz, D. M. 1990. Determinación de algunos aspectos biológicos de la grana o cochinilla del nopal (Dactylopius coccus Costa: Coccoidea: Dactylopiidae) en Chapingo, México. Tesis, Universidad Autónoma Chapingo, Chapingo, Estado de México. 71 p.

Cook, L. G. y P. J. Gullan. 2004. The gall-inducing habit has evolved multiple times among the eriococcid scale insects (Sternorrhyncha: Coccoidea: Eriococcidae). Biological Journal of the Linnean Society 83: 441-452.

Davey, K. G. 1969. La reproducción de los insectos. Alambra, México, D.F. 106 p.

De Lotto, G. 1974. On the status and identity of the cochineal insects (Homoptera: Dactylopiidae). Journal of Entomology South Africa 37: 167-193.

Eisner, T. S., S. Nowicky, M. Gotees and J. Meinwald. 1980. Red cochineal dye (carmine acid). In Role Nature Science 208: 1039-1042.

Ferris, G. F, 1955. Atlas of the scale insects of North America: the families Aclerdidae, Asterolecaniidae, Conchaspididae Dactylopiidae and Lacciferidae. III.
Stanford University Press, Palo Alto, California. 233 p.

Flores, F. I. V.; D. M. Alviar y J. Vilca. 1985. Época de siembra de la cochinilla del carmín $D$. coccus Costa en la comunidad campesina de Santa Rosa de Huatatas, Ayacucho, Perú. In Resúmenes del 1er Congreso Nacional de Tuna y Cochinilla, Ayacucho, Perú, p. 3637.

Gilreath, E. M. y J. W. Smith. 1987. Bionomics of Dactylopius confusus (Homoptera: Dactylopiidae): exclusion and subsequent impact on Opuntia (Cactaceae). Environmental Entomology 17: 730-738.

Gullan, P. y L. Cook. 1998. Are cochineal insects Eriococcids? In VIIIth International Symposium on Scale Insect Studies, Wye, England p. 19.

Hosking, R. J. 1984. The effect of temperature on the population growth potential of the Dactylopius austrianus de Lotto (Homoptera: Dactylopiidae) on Opuntia aurantiaca. Journal Australian Entomological Society 23: 133-159.

Howell, J. O. y M. L. Williams. 1976. An annotated key to the families of the scale insects (Homoptera: Coccoidea) of America. North of Mexico, based on characteristics of the adult female. Annals of the Entomological Society of America 69: 181-189.

Kogan, M. 1990. Resistencia de la planta en el manejo de plagas. In Introducción al manejo de plagas de insectos. Limusa, México, D.F., p. 753

Lazos, V. R. 1987. Producción de grana o cochinilla Dactylopius coccus Costa bajo tres ambientes en Chapingo. México. Tesis, Universidad Autónoma Chapingo, Estado de México. 5-20 p.

Mann, J. 1969. Cactus feeding insect and mites. United States National Museum Bulletin 256: 137-150.

Marín, L. R. 1985. Factores que deben cuidarse en la producción de la cochinilla del carmín (Dactylopius coccus Costa) Homoptera:Dactylopiidae. Revista Peruana de Entomología 37: 115-120.

Marín, L. R. y V. F. Cisneros. 1977. Biología y morfología de la cochinilla del carmín (Dactylopius coccus Costa) Homoptera: Dactylopiidae. Revista Peruana de Entomología 29: 70-76.

Méndez, G. J. S. 1992. Tasas de supervivencia y reproducción de la grana-cochinilla Dactylopius coccus Costa (Homoptera: Dactylopiidae) en diferentes temperaturas. Tesis de maestría,. Colegio de Postgraduados, Chapingo, Estado de México. 113 p.

Méndez, G. J. S., G. P. Aquino y A. Q. Moreno. 1990. Utilización de cuatro formas de nopal (Opuntia spp.) como hospederos de la grana-cochinilla (Homoptera: Dactylopiidae: Dactylopius coccus Costa) en el municipio de Salinas, San Luis Potosí. In Resúmenes de la IV Reunión Nacional y II Congreso Internacional sobre el Conocimiento y Aprovechamiento del Nopal, México, p. 47

Miller, D. R. 1976. Family Dactylopiidae in syllabus for 
workshop on scale identification. Proceedings of the National Meeting of the Entomological Society of America, November, Hawaii. 68-86 p.

Montiel, R. L. 1992. Valoración del cultivo de la granacochinilla Dactylopius coccus Costa, utilizando diferentes sustratos y fotoperiodos. Tesis, Universidad Autónoma de Tlaxcala, Ixtlahuaca. 93 p.

Morán, V. C. y H. G. Zimmermann. 1991. Biological control of cactus weeds of minor importance in South Africa. Journal Agriculture Ecosystems and Environment 37: $107-114$

Nur, U. S., W. Brown y J. W. Bardsley. 1987. Evolution of chromosome number in mealybugs (Pseudococcidae: Homoptera). Genetica 71: 53-60.

Piña, L. I. 1977. La grana o cochinilla del nopal. Monografías 1. Publicaciones de los Laboratorios Nacionales de Fomento Industrial (LANFI), México, D.F. 55 p.

Santibañez, W. L. 1990. Ciclo biológico, cultivo y aprovechamiento de la cochinilla del nopal Dactylopius coccus Costa, en el municipio de Villa Ordaz, adscrito al centro coordinador indigenista zapoteco del Valle de Oaxaca. Informe del Servicio Social de la División de Ciencias Biológicas y de la Salud. Departamento del hombre y su ambiente. Universidad Autónoma Metropolitana, Unidad Xochimilco, México, D.F. 198 p.

SAS Institute, 1996. SAS/STAT user's guide. Ver. 6.12 SAS Institute, Cary, North Caroline.

Solís, A. J. F. 1993. Escamas (Homóptera: Coccoidea); descripción, morfología y técnica de montaje. Departamento de Parasitología Agrícola. Universidad Autónoma Chapingo. Estado de México. 34-36 p.

Trachtenberg, S. y A. M. Mayer. 1981. Composition and properties of Opuntia ficus-indica mucilage. Phytochemistry 20: 2665-2628.

Velasco, P. H. y G. F. García. 1988. Estudio del ciclo biológico de la cochinilla o grana del nopal. In Resúmenes del XXXIII Congreso Nacional de Entomología. Morelia, Michoacán, p. 101-102.

Vera, G. J., V. M. Pinto y J. C. López. 1997. Ecología de poblaciones de insectos. Universidad Autónoma Chapingo, Estado de México. 210 p.

Vigueras, G. A. L. y M. L. Portillo. 1997. Cultivo de la grana o cochinilla del nopal. In Suculentas mexicanas, Cactáceas. CONABIO-SEMARNAP-UNAM. CVS, México, D.F., p. 39-45.

Zimmermann, E. C. 1948. Insects of Hawaii, vol. 5. University of Hawaii Press, Honolulu U.P.H. 110 p. 